\title{
REVIEW
}

\section{Effect of fructose on markers of non-alcoholic fatty liver disease (NAFLD): a systematic review and meta-analysis of controlled feeding trials}

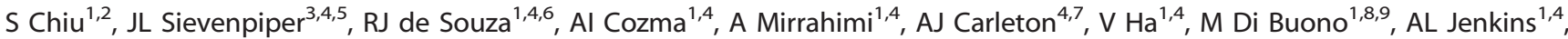 \\ LA Leiter ${ }^{1,4,10,11,12}$, TMS Wolever ${ }^{1,4,10,11,12}$, AC Don-Wauchope ${ }^{3,13}$, J Beyene ${ }^{6,14,15}$, CWC Kendall ${ }^{1,4,16}$ and DJA Jenkins ${ }^{1,4,10,11,12}$
}

BACKGROUND/OBJECTIVES: In the absence of consistent clinical evidence, there are concerns that fructose contributes to non-alcoholic fatty liver disease (NAFLD). To determine the effect of fructose on markers of NAFLD, we conducted a systematic review and meta-analysis of controlled feeding trials.

SUBJECTS/METHODS: We searched MEDLINE, EMBASE, CINAHL and the Cochrane Library (through 3 September 2013). We included relevant trials that involved a follow-up of $\geqslant 7$ days. Two reviewers independently extracted relevant data. Data were pooled by the generic inverse variance method using random effects models and expressed as standardized mean difference (SMD) for intrahepatocellular lipids (IHCL) and mean difference (MD) for alanine aminotransferase (ALT). Inter-study heterogeneity was assessed (Cochran $Q$ statistic) and quantified ( $I^{2}$ statistic).

RESULTS: Eligibility criteria were met by eight reports containing 13 trials in 260 healthy participants: seven isocaloric trials, in which fructose was exchanged isocalorically for other carbohydrates, and six hypercaloric trials, in which the diet was supplemented with excess energy ( $+21-35 \%$ energy) from high-dose fructose $(+104-220 \mathrm{~g} /$ day). Although there was no effect of fructose in isocaloric trials, fructose in hypercaloric trials increased both $\mathrm{IHCL}(\mathrm{SMD}=0.45(95 \%$ confidence interval $(\mathrm{Cl})$ : $0.18,0.72)$ ) and $\mathrm{ALT}(\mathrm{MD}=4.94 \mathrm{U} / \mathrm{l}(95 \% \mathrm{Cl}: 0.03,9.85))$.

LIMITATIONS: Few trials were available for inclusion, most of which were small, short ( $\leqslant 4$ weeks), and of poor quality. CONCLUSIONS: Isocaloric exchange of fructose for other carbohydrates does not induce NAFLD changes in healthy participants. Fructose providing excess energy at extreme doses, however, does raise IHCL and ALT, an effect that may be more attributable to excess energy than fructose. Larger, longer and higher-quality trials of the effect of fructose on histopathological NAFLD changes are required.

European Journal of Clinical Nutrition (2014) 68, 416-423; doi:10.1038/ejcn.2014.8; published online 26 February 2014

Keywords: sugars; non-alcoholic fatty liver disease; clinical trial; meta-analysis

\section{INTRODUCTION}

Non-alcoholic fatty liver disease (NAFLD) is the most prevalent chronic liver disease and a cause of raised liver enzymes in developed countries, ${ }^{1}$ affecting $10-30 \%$ of people in developed countries. $^{2,3}$ The increasing prevalence of NAFLD, which is closely linked with the increasing prevalence of obesity and type 2 diabetes mellitus (T2DM), ${ }^{2}$ has been associated with increased cardiovascular morbidity and mortality. ${ }^{1}$

Dietary factors that influence NAFLD have become a focus of attention. In particular, recent concerns have been raised regarding the role of dietary fructose in inducing NAFLD. ${ }^{4-7}$ Animal models featuring extreme levels of fructose exposure ${ }^{8-11}$

${ }^{1}$ Department of Nutritional Sciences, Faculty of Medicine, University of Toronto, Toronto, ON, Canada; ${ }^{2}$ Department of Human Biology, Faculty of Medicine, University of Toronto, Toronto, ON, Canada; ${ }^{3}$ Department of Pathology and Molecular Medicine, Faculty of Health Sciences, Hamilton, ON, Canada; ${ }^{4}$ Toronto 3D Knowledge Synthesis and Clinical Trials Unit, Clinical Nutrition and Risk Factor Modification Centre, St Michael's Hospital, Toronto, ON, Canada; ${ }^{5}$ The Li Ka Shing Knowledge Institute, St. Michael's Hospital, Toronto, ON, Canada; ${ }^{6}$ Department of Clinical Epidemiology \& Biostatistics, Faculty of Health Sciences, McMaster University, Hamilton, ON, Canada; ${ }^{7}$ Department of Undergraduate Medical Education (MD Program), Faculty of Medicine, University of Toronto, Toronto, ON, Canada; ${ }^{8}$ Heart and Stroke Foundation of Ontario, Toronto, ON, Canada; ${ }^{9}$ American Heart Association, Dallas, TX, USA; ${ }^{10}$ Keenan Research Center of the Li Ka Shing Knowledge Institute, St. Michael's Hospital, Toronto, ON, Canada; ${ }^{11}$ Division of Endocrinology, St Michael's Hospital, Toronto, ON, Canada; ${ }^{12}$ Department of Medicine, Faculty of Medicine, University of Toronto, Toronto, ON, Canada; ${ }^{13}$ Division of Clinical Chemistry and Immunology, Hamilton Regional Laboratory Medicine Program, Hamilton, ON, Canada; ${ }^{14}$ The Dalla Lana School of Public Health, Faculty of Medicine, University of Toronto, Toronto, ON, Canada;

${ }^{15}$ Child Health Evaluative Sciences (CHES), The Hospital for Sick Children Research Institute, Toronto, ON, Canada and ${ }^{16}$ College of Pharmacy and Nutrition, University of Saskatchewan, Saskatoon, SK, Canada. Correspondence: Dr JL Sievenpiper, Toronto 3D Knowledge Synthesis and Clinical Trials Unit, Clinical Nutrition and Risk Factor Modification Centre, St Michael's Hospital, \#6137-61 Queen Street East, Toronto, ON, Canada M5C 2T2.

E-mail: john.sievenpiper@utoronto.ca

Guarantors: JL Sievenpiper and DJA Jenkins.

Contributors: JLS, RJdS, AM, AJC, MDB, ALJ, LAL, TMSW, ACDW, JB, CWCK and DJAJ contributed to the conception and design. SC, JLS, RJdS, AIC and VH contributed to analysis and interpretation of the data. SC and JLS contributed to drafting of the article. JLS, RJdS, AIC, A Mirrahimi, AJC, VH, MDB, ALJ, LAL, TMSW, ACDW, JB, CWCK and DJAJ Contributed to critical revision of the article for important intellectual content. SC, JLS, RJdS, AIC, AM, AJC, VH, MDB, ALJ, LAL, TMSW, ACDW, JB, CWCK and DJAJ contributed to the final approval of the article. RJdS and JB contributed to statistical analyses. JLS, RJdS, AM, AJC, MDDB, ALJ, LAL, TMSW, JB, CWCK and DJAJ obtained the funding. AM and CWCK contributed to administrative, technical or logistic support. SC, AIC, JLS and RJdS contributed to collection and assembly of data.

Received 27 February 2013; revised 12 October 2013; accepted 12 November 2013; published online 26 February 2014 
and poor-quality observational studies ${ }^{12-14}$ have been used to underpin this hypothesis. In the absence of consistent clinical evidence, it is unclear whether fructose at typical levels of exposure induces NAFLD. To determine the effect of fructose on markers of NAFLD in humans, we conducted a systematic review and meta-analysis of available controlled feeding trials.

\section{SUBJECTS AND METHODS}

Design

We followed the Cochrane Handbook for Systematic Reviews of Interventions ${ }^{15}$ and the Preferred Reporting Items for Systematic Reviews and Meta-Analysis guidelines. ${ }^{16}$ The review protocol is available at ClinicalTrials.gov (registration number: NCT01363791).

\section{Study selection}

We searched MEDLINE, EMBASE, CINAHL and the Cochrane Library databases through 3 September 2013 for relevant articles. Supplementary Appendix Table 1 shows the full search term used in this study. Manual searches supplemented the electronic search strategy. No restrictions were placed on language. We included controlled trials investigating the effect of oral fructose on markers of NAFLD. A comparison was considered isocaloric when the carbohydrate comparator was exchanged for an equal amount of fructose. If the trial involved overfeeding of fructose so that the fructose provided excess energy resulting in a positive energy balance, then the comparison was still considered isocaloric as long as the carbohydrate comparator was matched for the excess energy resulting in the same positive energy balance. A comparison was considered hypercaloric when a control diet was supplemented with excess energy from fructose compared with the same control diet alone without the excess energy. Trials that involved a follow-up of $<7$ days follow-up, administered intravenous fructose, lacked a control diet or did not provide suitable endpoint data were excluded.

\section{Data extraction}

Two reviewers (SC, AIC) independently reviewed and extracted relevant data from each report. The quality of each study was assessed using the Heyland methodological quality score (MQS). ${ }^{17}$ Disagreements were reconciled by consensus. Mean \pm s.d. differences between fructose and control arms were extracted as the main end points. In those trials where the data were included in figures and not provided numerically, we used software program Plot Digitizer (http://plotdigitizer.sourceforge.net/) to extract the data. Additional information was requested from the authors of all included trials.

\section{Access to study}

All authors had access to the study data and reviewed and approved the final manuscript.

\section{Statistical analysis}

Data analyses were conducted using Review Manager (RevMan) version 5.1.6 (Copenhagen, Denmark) for primary analyses and Stata (version 12, College Station, TX, USA) for subgroup analyses. Separate analyses were conducted for the isocaloric and hypercaloric trials using the generic inverse variance method with random effects weighting. Data were expressed as standardized mean differences with $95 \%$ confidence intervals (Cls) for intrahepatocellular lipid (IHCL) and mean differences (MD) with 95\% Cls for alanine aminotransferase (ALT).

Trials that did not report SE values had these computed from the available statistics using standard formulae. ${ }^{15,18}$ To generate SE for included crossover trials, we assumed a paired analyses as described by Elbourne. ${ }^{18}$ If insufficient data were available for computations in crossover trials, SE values were imputed using the pooled correlation coefficient between baseline and end-of-study values derived from a meta-analysis of trials reporting sufficient data or assuming a conservative correlation coefficient of 0.5 with sensitivity analyses at 0.25 and 0.75 .

Inter-trial heterogeneity was assessed by the Cochran $Q$ statistic with $\alpha<0.10$ considered significant, and quantified by the $P^{2}$ statistic, where $P^{2} \geqslant 50 \%$ indicates substantial heterogeneity. ${ }^{15}$ Sources of heterogeneity were investigated by sensitivity analyses in which each individual study was removed from the analysis and through a priori subgroup analyses by comparator, baseline values, fructose form, follow-up, MQS, randomization, design and energy balance. Meta-regression analyses assessed the significance of subgroup effects. Publication bias was evaluated via visual inspection of funnel plots and Egger and Begg tests.

\section{RESULTS}

Search results

Figure 1 shows the trial selection process. We identified 1437 eligible reports. A total of eight reports (providing data for 13 trials) were selected for analyses. ${ }^{19-26}$

\section{Trial characteristics}

Table 1 shows the trial characteristics. Only two markers of NAFLD were identified: $I H C L$ and ALT. None of the available trials assessed NAFLD histologically from liver biopsies. There were a total of seven isocaloric trials (four for $\mathrm{HCL}$ and six for ALT) in 184 healthy participants, and six hypercaloric trials (five for IHCL and four for ALT) in 76 participants $(n=60$ healthy and $n=16$ offspring of type 2 diabetes). The majority of both sets of trials were conducted in European countries in an outpatient setting and tended to be small (median (interquartile range (IQR)) sample size, 29.0 (24.5-31.5) and 13.5 (10.5-15.8), in isocaloric and hypercaloric trials, respectively).

Participants tended to be healthy, young (median (IQR) age $=30.5$ years $(26.3-33.9$ years) and 27.6 years $(24.7-33.9$ years)), male (median (IQR) percent male:female ratio $=100 \%$ (56-100) and 100\% (77.5-100)), and overweight (median (IQR) body mass index $=25.9 \mathrm{~kg} / \mathrm{m}^{2}\left(22.4-29.4 \mathrm{~kg} / \mathrm{m}^{2}\right)$ and $24.3 \mathrm{~kg} / \mathrm{m}^{2}$ $\left.\left(22.2-28.5 \mathrm{~kg} / \mathrm{m}^{2}\right)\right)$ in isocaloric and hypercaloric trials, respectively. Median (IQR) baseline ALAT values (in U/I) were 26.0 (23-28.9) in isocaloric trials and 20.58 (18.5-25.8) in hypercaloric trials. Median values for baseline $\mathrm{IHCL}$ (in \%) could not be computed from the data reported.

Crossover designs were used in $29 \%$ of isocaloric trials and all hypercaloric trials. The majority of isocaloric trials (86\%) and $17 \%$ of hypercaloric trials were randomized. Glucose was the comparator in all isocaloric trials except in the two trials of Aeberli et al., ${ }^{21}$ where glucose and sucrose were the comparators in the high dose trial and glucose and starch were the comparators in the low dose trial. The control diet alone without the added energy from fructose was the comparator in all hypercaloric trials. Although comparisons in all isocaloric trials were matched for energy, $86 \%$ of the isocaloric trials provided fructose and the carbohydrate comparator under conditions of positive energy balance (that is, both arms provided excess energy), whereas $14 \%$ provided fructose and the carbohydrate comparator under conditions of neutral energy balance (that is, both arms provided energy to maintain weight). All isocaloric and hypercaloric trials administered fructose in fluid form at a median (IQR) dose of $182 \mathrm{~g} /$ day $(115-204 \mathrm{~g} /$ day) for isocaloric trials and $+193 \mathrm{~g} /$ day $(+158-211 \mathrm{~g} /$ day $)$ for hypercaloric trials. The median (IQR) excess energy provided by the hypercaloric trials was $+25 \%(+23-33 \%)$. All isocaloric and hypercaloric trials featured high-carbohydrate and low-fat diets with similar macronutrient profiles: $50-55 \%$ energy carbohydrate, 30-35\% energy fat and $13-15 \%$ energy protein. Metabolic feeding control was used in $14 \%$ of isocaloric trials and $17 \%$ of hypercaloric trials; partial-metabolic feeding control was used in 43 and $17 \%$ and the remainder provided fructose as a supplement. The median (IQR) dietary follow-up was 4 weeks (3-8 weeks) for isocaloric trials and 3 weeks (1.25-4 weeks) for hypercaloric trials.

The Heyland MQS was considered high (MQS $\geqslant 8$ ) in $71 \%$ of isocaloric and $33 \%$ of hypercaloric trials. Lack of or poor description of randomization, nonconsecutive or poorly described patient selection and absence of double-blinding contributed to lower scores. Funding of all trials was from a combination of agency alone (69\%) or agency-industry sources (31\%). None were 
1437 Reports Identified (to September 3, 2013) 329 CINAHL (to September 3, 2013) 195 Cochrane Library (to September 3, 2013)

553 EMBASE (to September 3, 2013)

355 MEDLINE (to September 3, 2013) 5 Manual Searches

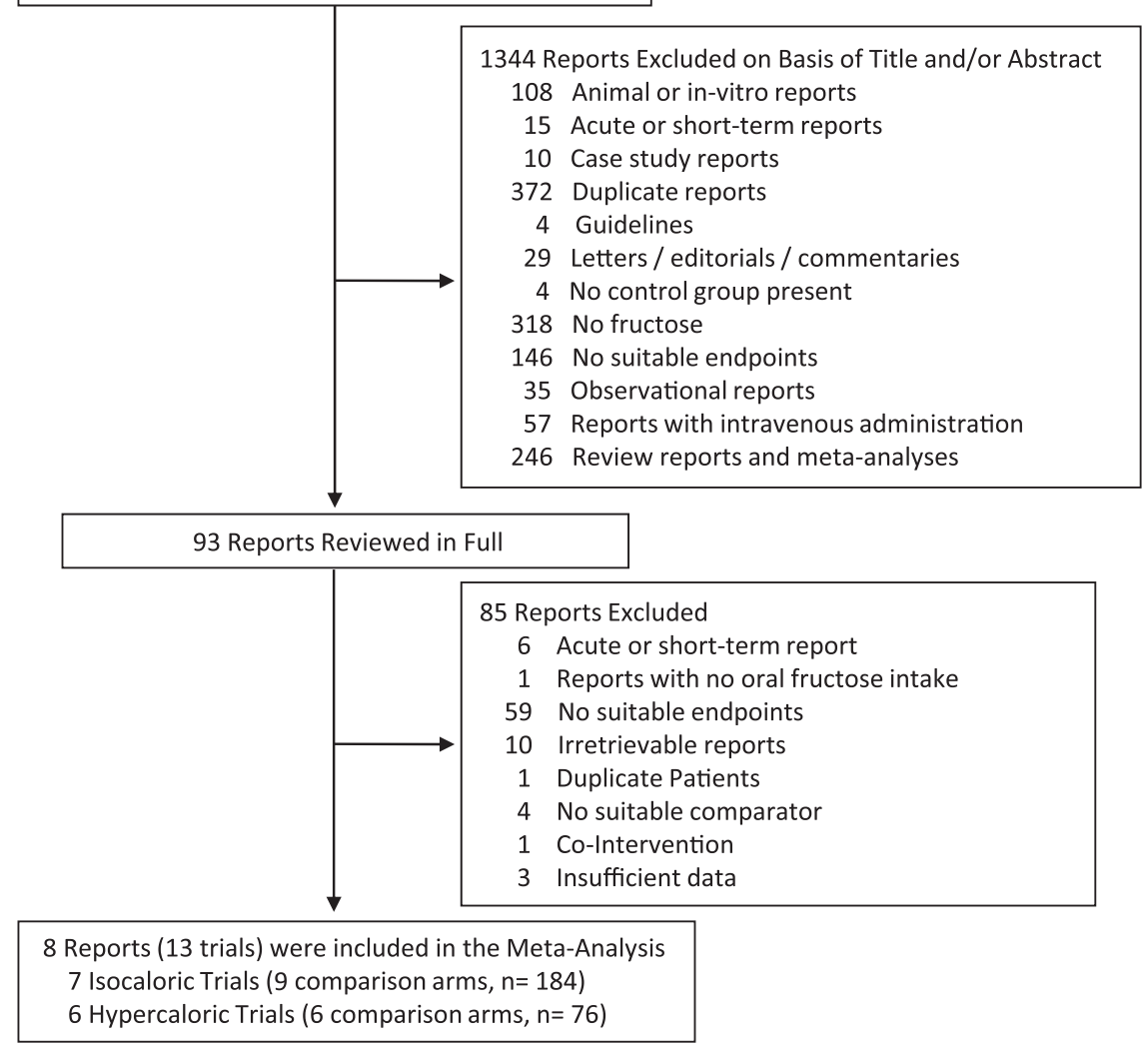

Figure 1. Flow of the literature.

funded by industry alone. None reported a potential conflict of interest.

Effect of fructose on $\mathrm{IHCL}$

Figure 2a shows the effect of fructose on $\mathrm{IHCL}$ in isocaloric trials. Primary pooled analyses showed no effect of fructose on $\mathrm{IHCL}$ (standardized mean difference $=-0.09(95 \% \mathrm{Cl}$ : $-0.36-0.18)$, $P=0.51$ ), with no evidence of inter-study heterogeneity $\left(I^{2}=0 \%, P=0.95\right)$. Sensitivity analyses did not alter the results. Meta-regression analyses showed no statistically significant subgroup effects (Supplementary Appendix Figure 1).

Figure $2 \mathrm{~b}$ shows the effect of fructose on IHCL in hypercaloric trials. Primary pooled analyses showed that fructose raised $\mathrm{IHCL}$ (standardized mean difference $=0.45 \quad(95 \% \quad \mathrm{Cl}:$ 0.18-0.72), $P=0.001)$, though there was significant inter-study heterogeneity $\left(I^{2}=55 \%, P=0.07\right)$. Sensitivity analyses did not alter the results, but identified that the removal of Lê et al., ${ }^{23}$ a study conducted in offspring of individuals with T2DM, eliminated evidence of inter-study heterogeneity $\left(I^{2}=0 \%, P=0.87\right)$. Meta-regression analyses showed no statistically significant subgroup effects (Supplementary Appendix Figure 2), and inter-study heterogeneity remained largely unexplained.

Effect of fructose on liver enzymes

Figure 3 a shows the effect of fructose on ALT in isocaloric trials. To approximate paired analyses for crossover trials, we used a conservative correlation coefficient of 0.5 . Primary pooled analyses showed no effect of fructose on ALT (MD $=0.15$ (95\% Cl: -1.51 to 1.82), $P=0.86)$, with no significant evidence of inter-study heterogeneity $\left(I^{2}=0 \%, P=0.97\right)$. Neither sensitivity analyses nor the use of more $(0.75)$ or less $(0.25)$ conservative correlation coefficients altered the results. Meta-regression analyses revealed no statistically significant subgroup effects (Supplementary Appendix Figure 3).

Figure $3 \mathrm{~b}$ shows the effect of fructose on ALT in hypercaloric trials. To approximate paired analyses for crossover trials, we needed to use a conservative correlation coefficient of 0.5 . Primary analyses showed a significant ALT-increasing effect $(\mathrm{MD}=4.94$ (95\% Cl: 0.03-9.85), $P=0.05$ ), with significant evidence of interstudy heterogeneity $\left(I^{2}=78 \%, P=0.003\right)$. Sensitivity analyses revealed the removal of either Lê et al., ${ }^{23}$ Sobrecases et al. ${ }^{24}$ or Johnston et al. ${ }^{26}$ led to a loss of significance (MD $=2.97(95 \% \mathrm{Cl}$ : $-1.40,7.35) ; \mathrm{MD}=5.40(95 \% \mathrm{Cl}:-1.97,12.78)$; and $\mathrm{MD}=4.84$ $(95 \% \mathrm{Cl}:-1.51,11.19)$ ), respectively. Removal of Cox et al., ${ }^{25}$ significantly reduced evidence of inter-study heterogeneity $\left(I^{2}=56 \%, P=0.11\right)$. Sensitivity analysis using higher $(0.75)$ or lower (0.25) conservative correlation coefficients did not alter the results. Meta-regression analyses did not show any statistically significant subgroup effects (Supplementary Appendix Figure 4).

\section{Publication bias}

We examined funnel plots for evidence of publication bias (Supplementary Appendix Figure 5). There was some evidence of slight asymmetry in the hypercaloric trials for ALT on visual 


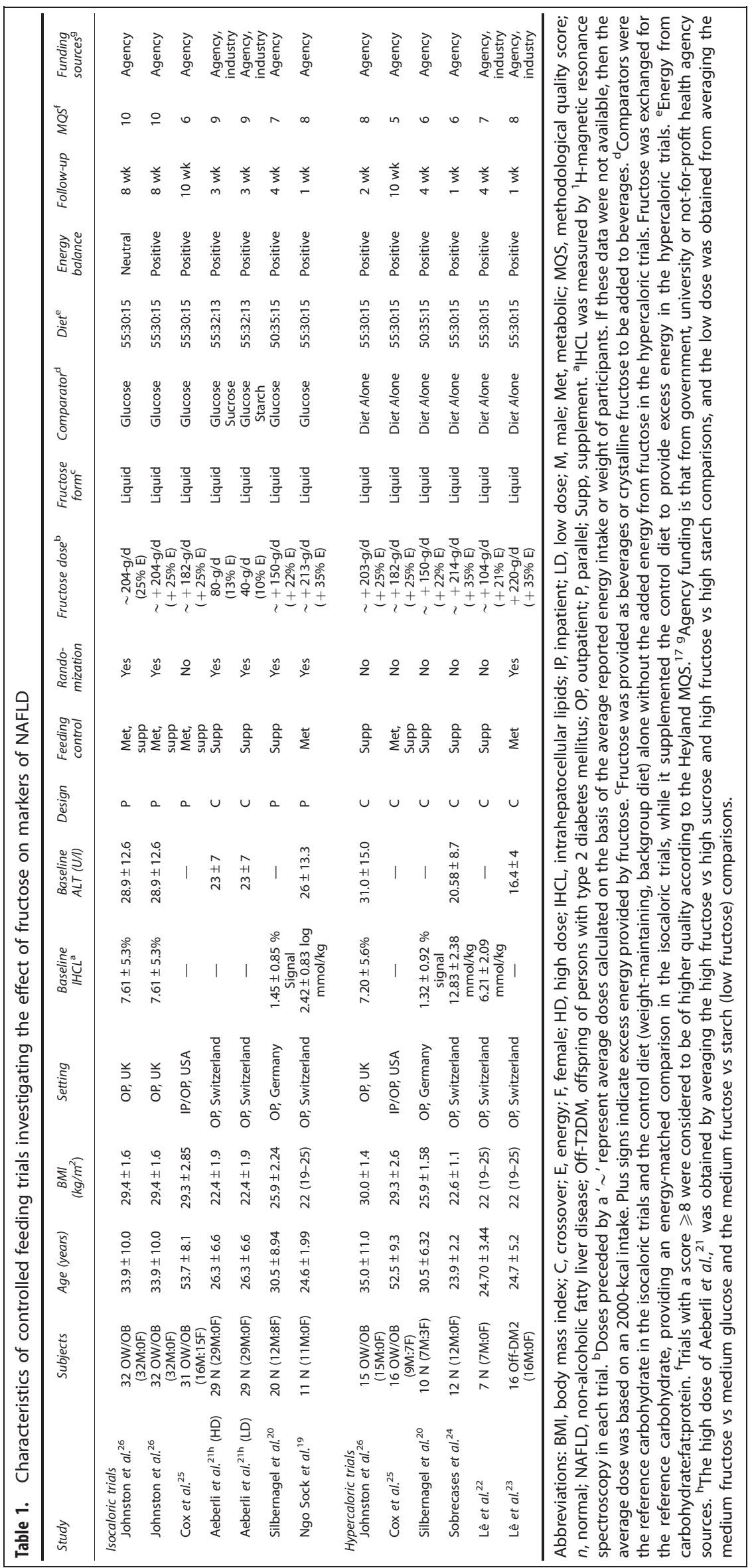


a Isocaloric Trials

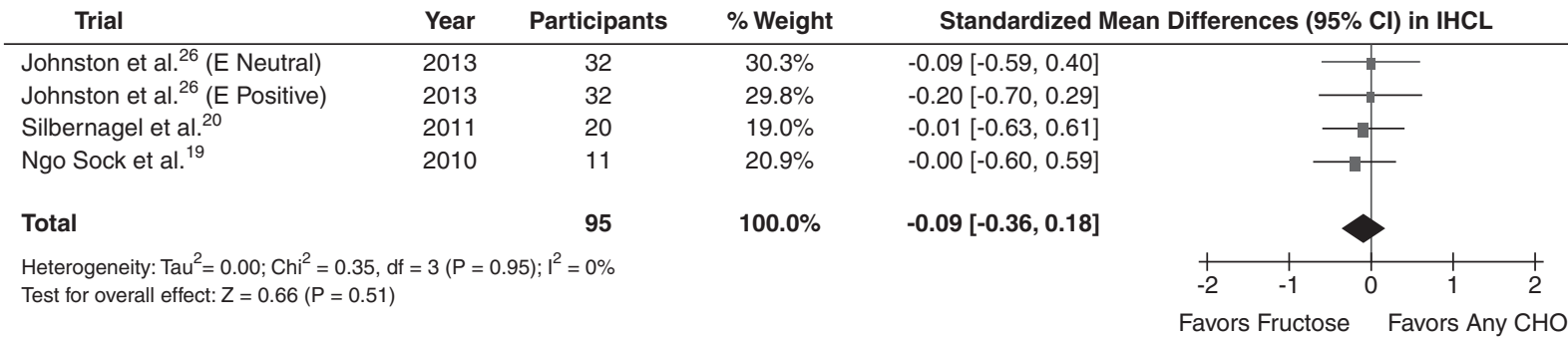

b Hypercaloric Trials

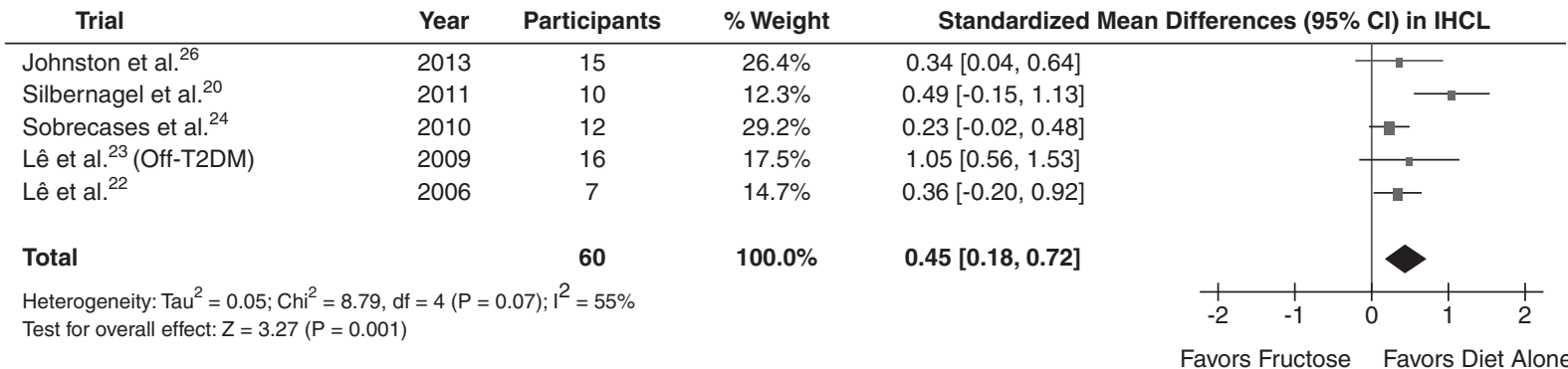

Figure 2. Forest plots of the effect of fructose on intrahepatocellular lipid (IHCL) in healthy participants in (a) isocaloric and (b) hypercaloric feeding trials. Pooled effect estimates shown as diamonds. Data are expressed as weighted MD with $95 \% \mathrm{Cl}$ using generic inverse variance random effects models. Inter-study heterogeneity was tested by Cochrane's $Q$ statistic $\left(\chi^{2}\right.$-test) at a significance level of $P<0.10$ and quantified by $I^{2}$, where $I^{2} \geqslant 50 \%$ is considered to be evidence of substantial heterogeneity and $\geqslant 75 \%$, considerable heterogeneity. Any CHO denotes any carbohydrate comparator; E neutral, neutral energy balance; E positive, positive energy balance; and Off-T2DM, offspring of T2DM.

a Isocaloric Trials

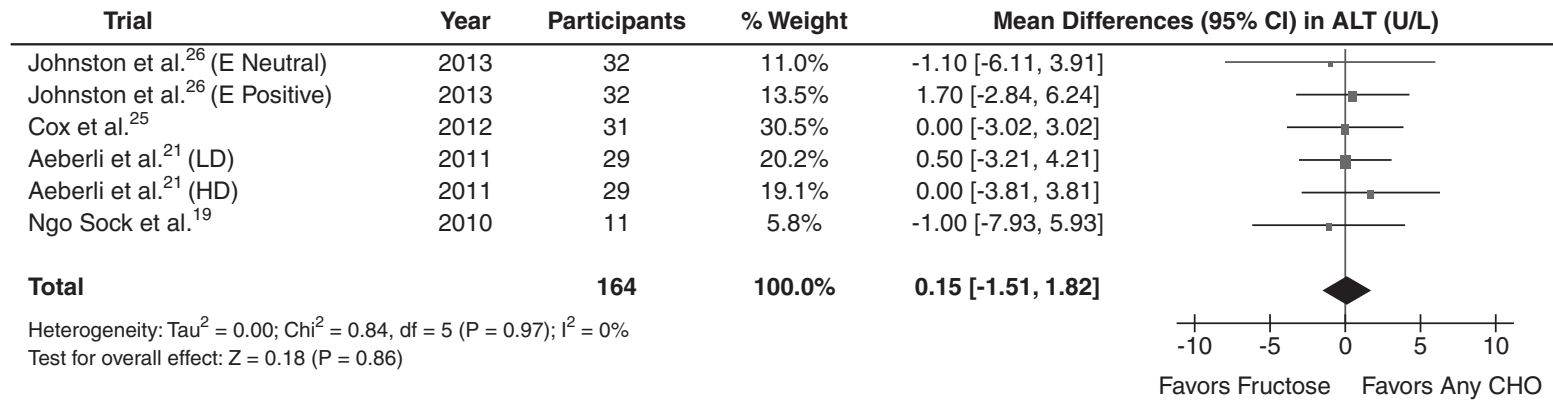

b Hypercaloric Trials

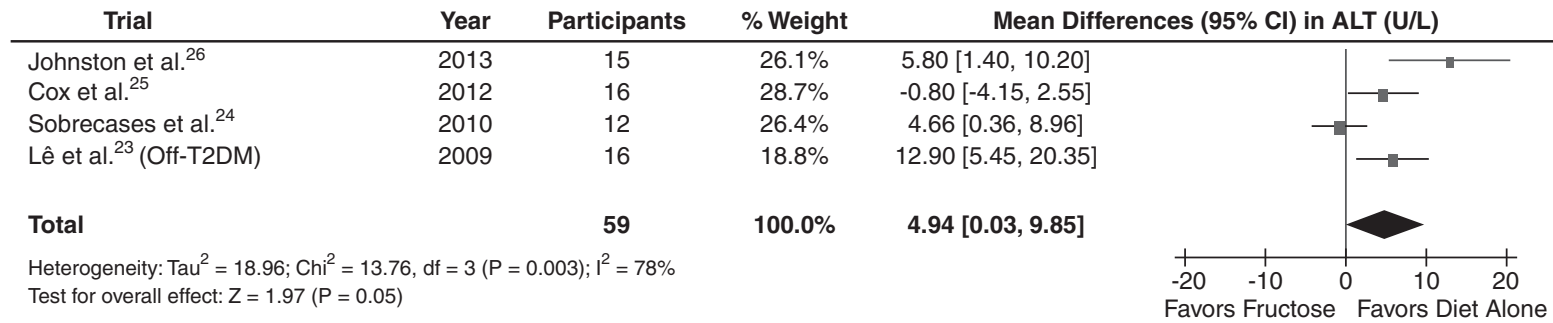

Figure 3. Forest plots of the effect of fructose on ALT in healthy participants in (a) isocaloric and (b) hypercaloric feeding trials. Pooled effect estimates shown as diamonds. Data are expressed as weighted MD with $95 \% \mathrm{Cl}$ using generic inverse variance random effects models. Inter-study heterogeneity was tested by Cochrane's Q statistic ( $\chi^{2}$-test) at a significance level of $P<0.10$ and quantified by $I^{2}$, where $I^{2} \geqslant 50 \%$ is considered to be evidence of substantial heterogeneity and $\geqslant 75 \%$, considerable heterogeneity. Any $\mathrm{CHO}$ denotes any carbohydrate comparator; $\mathrm{E}$ neutral, neutral energy balance; $E$ positive, positive energy balance; HD, high dose; LD, low dose; and Off-T2DM, offspring of T2DM.

inspection ( $P=0.056$ by Egger test; $P=0.089$ by Begg test), but no small study effects were detected among the isocaloric and hypercaloric trials for either $\mathrm{HCL}$ or ALT by Egger and Begg tests $(P>0.05)$.

\section{DISCUSSION}

The present aggregate analyses of 13 trials in 260 predominantly young, male participants, who were overweight/obese or otherwise healthy, investigated the effect of fructose on markers of 
NAFLD under two different types of trial conditions: one where fructose in beverage form was isocalorically exchanged for other carbohydrates and the other where fructose in beverage form supplemented control diets with excess energy ( $+21-35 \%$ energy) at extreme doses (104-220 g/day) relative to the same control diets without the excess energy. These two types of trial conditions produced different results. Although there was no effect of fructose in isocaloric trials, fructose increased both $\mathrm{IHCL}$ and ALT in hypercaloric trials.

\section{Relation of findings to other lines of evidence}

Our finding of a lack of effect of fructose on NAFLD markers in isocaloric trials contradicts evidence from animal models and observational studies. The ability of fructose to induce a metabolic syndrome phenotype and NAFLD is thought to lie in its ability to act as an unregulated substrate for de novo lipogenesis, bypassing the major rate-limiting step of glycolysis at phosphofructokinase. ${ }^{7,27}$ This mechanism contributes significantly to de novo lipogenesis in rodent models, in which fructose fed at supraphysiological doses under isocaloric ( $\sim 60 \%$ energy) or hypercaloric ( $+30 \%$ excess energy) conditions induces steatosis and steatohepatitis. ${ }^{8-11}$ Small cross-sectional and retrospective case-control studies have also shown an association between fructose-containing sugar intake and NAFLD. ${ }^{12-14}$ Clinical translation of these data, however, has several limitations. Rodent models are complicated by supraphysiological doses and excess energy, ${ }^{28}$ and marked differences exist in the metabolic fate of fructose between animals and humans. Although de novo lipogenesis from fructose accounts for $60-70 \%$ of fatty acids in rodents, ${ }^{28}$ its contribution in humans is quantitatively insignificant. $^{29,30}$ Two carefully conducted reviews of the available isotopic tracer studies showed that de novo lipogenesis from fructose contributes $<1 \%$ of fatty acids, whereas glucose $(\sim 50 \%)$, lactate $(\sim 25 \%)$ and glycogenesis ( $>15 \%)$ synthesis remain the major pathways of hepatic fructose disposal in humans. ${ }^{29,30}$ Cross-sectional and retrospective case-control studies do not provide evidence of causation and have found positive associations with many other factors that might be equal or better predictors of NAFLD, such as increased intake of energy, total fat, total carbohydrate, animal protein, cholesterol and the $n-6: n-3$ ratio of polyunsaturated fatty acids and decreased intake of dietary fiber. ${ }^{6}$ No large prospective observational studies have evaluated the relationship between fructose and NAFLD.

Energy represents an important confounding factor in the effect of fructose. Overfeeding of a 'fast food' diet has been shown to relate to an increase in ALT in healthy paticipants. ${ }^{31}$ Randomized trials of energy-restricted diets focusing on total energy reduction and exercise to promote weight loss have also shown reversal of NAFLD markers in people with NAFLD. ${ }^{6,32}$ In the present analyses, we observed increases in $\mathrm{HCL}$ and ALT only in hypercaloric trials. The lack of effect in the isocaloric trials was seen even under conditions of positive energy balance. Six of the isocaloric trials (three of four trials assessing $\mathrm{IHCL}^{19,20,26}$ and five of six trials assessing $A L T^{19,25,26}$ ) used excess energy diets in both the fructose and comparator arms, so permitting the effect of fructose to be isolated from that of energy under matched, yet excess energyfeeding conditions. Restricting our analyses to these trials did not show an effect of fructose on NAFLD markers. We made similar observations for the lack of effect of fructose on both body weight $^{33}$ and uric acid ${ }^{34}$ in two earlier systematic reviews and meta-analyses. These data suggest that the effect of fructose on NAFLD markers may not be different from that of other carbohydrates as long as energy remains matched.

Previous meta-analyses have identified subgroup effects on related metabolic end points. A dose threshold was observed for a triglyceride-raising effect of fructose: $\geqslant 100 \mathrm{~g} /$ day for fasting and $\geqslant 50 \mathrm{~g} /$ day for postprandial triglycerides across different participant groups ${ }^{35}$ and $>60 \mathrm{~g} /$ day for fasting triglycerides in type 2 diabetes. ${ }^{36}$ A fasting triglyceride-raising effect of fructose was also seen where starch was the comparator and follow-up was $\leqslant 4$ weeks in type 2 diabetes, ${ }^{36}$ whereas a weight-loss effect of fructose was seen in overweight/obese individuals and where fructose was in fruit form. ${ }^{33}$ None of these subgroup analyses were significant in the present analysis. Although the number of trials was small, the lack of effect modification across a priori subgroup analyses was consistent with that seen in our earlier meta-analyses for blood pressure ${ }^{37}$ and uric acid. ${ }^{34}$

\section{Limitations}

Our analyses have several limitations. First, the available trials had small sample sizes and narrow participant demographics. Combining the seven isocaloric and six hypercaloric trials, our median sample size was 16 participants, the majority of whom were young, male, and either overweight/obese (without any comorbidities) or otherwise healthy. Although the baseline IHCL values in the overweight/obese participants were $>95$ th percentile for the general population $(>5.56 \%){ }^{38}$ the data generated from such a generally healthy group may not be truly reflective of the disease physiology in people with or at risk for NAFLD, especially given that in patients with histologically established NAFLD, fructose may be associated with worse disease. ${ }^{39}$ Second, none of the trials in our meta-analysis had a follow-up period exceeding 10 weeks. The isocaloric and hypercaloric trials had a median follow-up of 4 weeks and 3 weeks, respectively. It is unclear whether the changes in IHCL and ALT seen in hypercaloric trials or the null effects seen in isocaloric trials are sustainable over the longer term. Third, study quality was poor (MQS $<8$ ) in $46 \%$ of the trials. Most of the low-quality scores were attributable to a lack of or poor description of randomization, nonconsecutive or poorly described patient selection and absence of blinding. However, no effect modification by study quality was seen in subgroup analyses. Fourth, none of the available trials assessed NAFLD by histological analysis of liver biopsies. This analysis remains the gold standard assessment for NAFLD, as ALT is quite insensitive, while IHCL by ${ }^{1} \mathrm{H}$-magnetic resonance spectroscopy cannot detect inflammation and/or fibrosis. ${ }^{1}$ The two measurements, however, showed good agreement among the trials. Finally, given the small number of available trials, publication bias remains unclear, although no small study effects were detected.

\section{Implications}

Although our results bear on the question of whether fructosecontaining sugar-sweetened beverages have a unique role in the development of NAFLD, their translation to 'real-world' intake patterns is complicated. The median level of fructose exposure was $>95$ th percentile U.S. intake $(87 \mathrm{~g} / \text { day })^{40}$ across all trials: 2.5 -fold greater than this threshold $(+215 \mathrm{~g} /$ day providing $+35 \%$ excess energy) in the hypercaloric trials, in which there was an effect, and 1.4-fold greater than this threshold ( $115 \mathrm{~g} /$ day) in the isoclaloric trials, in which there was no effect. Also, no trials used non-beverage grain or fruit sources of fructose, which together account for $>30 \%$ of fructose in the U.S. diet ${ }^{40}$ and have been linked (as whole grains and fruits) to weight loss and improved metabolic outcomes in large prospective cohort studies ${ }^{41,42}$ and randomized trials. ${ }^{43,44}$ Dietary trials of more representative sources of fructose at more representative levels of exposures remain a research priority.

\section{CONCLUSIONS}

In conclusion, our preliminary systematic review and meta-analysis does not support a NAFLD-inducing effect of fructose in isocaloric exchange for other carbohydrates at levels of exposure that are 
well above that found in Western diets. The evidence does support an IHCL- and ALT-increasing effect of diets supplemented with fructose providing excess energy $(+21-35 \%$ energy) at extreme doses (104-220 g/day). Confounding from excess energy, however, cannot be excluded in the hypercaloric trials, such that the observed NAFLD-inducing effect is more attributable to the excess energy than the fructose itself. Other sources of uncertainty in our analyses include the small number of available trials (four for IHCL and six for ALT), as well as the relatively small sample sizes ( $<30$ participants/trial) and narrow participant demographics (most participants were young and relatively healthy). The short follow-up (all trials were <12-weeks) using $\mathrm{IHCL}$ and ALT as markers of NAFLD may also not be relevant to the natural history of NAFLD over the longer term, especially in people who may be at low risk. It is unclear whether a larger number of trials which address these many issues will show the same findings when analyzed collectively. To understand the role of fructose in the epidemic of NAFLD, there remains a need for larger, longer, highquality trials of the effect of 'real-world' intake patterns of fructose on histopathological changes of NAFLD in at risk populations.

\section{CONFLICT OF INTEREST}

JLS has received research support from the Canadian Institutes of Health Research $(\mathrm{ClHR})$, Calorie Control Council, The Coca-Cola Company (investigator initiated unrestricted grant), Pulse Canada and The International Tree Nut Council Nutrition Research \& Education Foundation. He has received travel funding, speaker fees and/ or honoraria from the American Heart Association (AHA), American College of Physicians (ACP), American Society for Nutrition (ASN), National Institute of Diabetes and Digestive and Kidney Diseases (NIDDK) of the National Institutes of Health (NIH), Canadian Diabetes Association (CDA), Canadian Nutrition Society (CNS), Calorie Control Council, Diabetes and Nutrition Study Group (DNSG) of the European Association for the Study of Diabetes (EASD), International Life Sciences Institute (ILSI) North America, International Life Sciences Institute (ILSI) Brazil, University of South Carolina, Calorie Control Council, Canadian Sugar Institute, Abbott Laboratories, Pulse Canada, Dr Pepper Snapple Group, and The Coca-Cola Company. He is on the Clinical Practice Guidelines Expert Committee for Nutrition Therapy of both the Canadian Diabetes Association (CDA) and European Association for the study of Diabetes (EASD), as well as being on the American Society for Nutrition (ASN) writing panel for a scientific statement on the metabolic and nutritional effects of fructose, sucrose and high fructose corn sirup. He is a member of the Transcultural Diabetes Algorithm (tDNA) Collaborative Group and the International Carbohydrate Quality Consortium (ICQC). He is an unpaid scientific advisor for the International Life Science Institute (ILSI) North America, Food, Nutrition, and Safety Program (FNSP). His wife is an employee of Unilever Canada. RJdS has received research support from the CIHR Calorie Control Council, the Canadian Foundation for Dietetic Research (CFDR), and The Coca-Cola Company (investigator initiated, unrestricted grant). He has served as an external resource person to the World Health Organization's (WHO) Nutrition Guidelines Advisory Group (NUGAG) and was the lead author of a systematic review and meta-analysis commissioned by the WHO of trans-fatty acids and health outcomes. The WHO paid for his travel and accommodation to attend NUGAG Meetings in Hangzhou, China and Copenhagen, Denmark. AIC and VH received a travel award to attend the 'Journey Through Science Day' hosted by PepsiCo and the New York Academy of Sciences (NYAS). VH has also received research support from the CIHR and WHO for work on a systematic review and meta-analysis commissioned by the $\mathrm{WHO}$ of the relation of saturated fatty acids with health outcomes. AM and AJC have received research support from the CIHR. ALJ is a part owner, VicePresident, and Director of Research of Glycemic Index Laboratories, Toronto, Canada. She has received research support from the CDA. TMSW is a part owner and the President of Glycemic Index Laboratories, Toronto, Canada and has authored several popular diet books on the glycemic index for which he has received royalties from Phillipa Sandall Publishing Services and CABI Publishers. He has received consultant fees, honoraria, travel funding, or research support from or served on the scientific advisory board for CIHR, CDA Dairy Farmers of Canada, McCain Foods, Temasek Polytechnic, Northwestern University, Royal Society of London, Glycemic Index Symbol program, CreaNutrition AG, McMaster University, Canadian Society for Nutritional Sciences, National Sports and Conditioning Association, Faculty of Public Health and Nutrition-Autonomous University of Nuevo Leon, Diabetes and Nutrition Study Group of the European Association for the Study of Diabetes. JB has received research support from the CIHR, Calorie Control Council and The Coca-Cola Company (investigator initiated, unrestricted). CWCK has received consultant fees, honoraria, travel funding, or research support from or served on the scientific advisory board for the CIHR, Calorie Control Council, The Coca-Cola Company (investigator initiated, unrestricted), Abbott Laboratories, Advanced Food Materials Network, Almond Board of California, American Peanut Council, American Pistachio Growers, Barilla, California Strawberry Commission, Canola Council of Canada, Danone, General Mills, Hain Celestial, International Tree Nut Council, Kellogg, Loblaw Brands Ltd, Oldways, Orafti, Paramount Farms, Pulse Canada, Saskatchewan Pulse Growers, Solae and Unilever. DJAJ has received consultant fees, honoraria, travel funding or research support from or served on the scientific advisory board for the CIHR, Canadian Foundation for Innovation (CFI), Ontario Research Fund (ORF), and Advanced Foods and Materia Network (AFMNet) Calorie Control Council, The Coca-Cola Company (investigator initiated, unrestricted), Barilla, Solae, Unilever, Hain Celestial, Loblaws Supermarkets Inc., Sanitarium Company, Herbalife International, Pacific Health Laboratories, Inc., Metagenics/MetaProteomics, Bayer Consumer Care, Oldways Preservation Trust, The International Tree Nut Council Nutrition Research \& Education, The Peanut Institute, Procter and Gamble Technical Center Limited, Griffin Hospital for the development of the NuVal System, Soy Advisory Board of Dean Foods, Alpro Soy Foundation, Nutritional Fundamentals for Health, Pacific Health Laboratories, Kellogg's, Quaker Oats, The Coca-Cola Sugar Advisory Board, Pepsi Company, Agrifoods and Agriculture Canada (AAFC), Canadian Agriculture Policy Institute (CAPI), The Almond Board of California, The California Strawberry Commission, Orafti, the Canola and Flax Councils of Canada, Pulse Canada, the Saskatchewan Pulse Growers, and Abbott Laboratories. The remaining authors declare no conflict of interest.

\section{ACKNOWLEDGEMENTS}

This work was funded by a Canadian Institutes of Health Research (CIHR) Knowledge Synthesis grant (funding reference number, 102078) and a research grant from the Calorie Control Council. RJdS was funded by a CIHR Postdoctoral Fellowship Award and AM was funded by a CIHR Canada Graduate Scholarship Master's award. VH and AIC were supported by Ontario Graduate Scholarships. AIC was also funded by Canadian Institutes of Health Research (CIHR) Fredrick Banting and Charles Best Canada Graduate Scholarship and Banting and Best Diabetes Centre (BBDC)-Novo Nordisk Studentship. DJAJ was funded by the Government of Canada through the Canada Research Chair Endowment. None of the sponsors had a role in any aspect of the present study, including design and conduct of the study; collection, management, analysis, and interpretation of the data; and preparation, review, or approval of the manuscript.

\section{REFERENCES}

1 Bhatia LS, Curzen NP, Calder PC, Byrne CD. Non-alcoholic fatty liver disease: a new and important cardiovascular risk factor? Eur Heart J 2012; 33: $1190-1200$

2 Lazo M, Clark JM. The epidemiology of nonalcoholic fatty liver disease: a global perspective. Semin Liver Dis 2008; 28: 339-350.

3 Lazo M, Hernaez R, Bonekamp S, Kamel IR, Brancati FL, Guallar E et al. Non-alcoholic fatty liver disease and mortality among US adults: prospective cohort study. BMJ 2011; 343: d6891.

4 Lustig RH, Schmidt LA, Brindis CD. Public health: the toxic truth about sugar. Nature 2012; 482: 27-29.

5 Lim JS, Mietus-Snyder M, Valente A, Schwarz JM, Lustig RH. The role of fructose in the pathogenesis of NAFLD and the metabolic syndrome. Nat Rev Gastroenterol Hepatol 2010; 7: 251-264.

6 Mouzaki M, Allard JP. The role of nutrients in the development, progression, and treatment of nonalcoholic fatty liver disease. J Clin Gastroenterol 2012; 46 457-467.

7 Dekker MJ, Su Q, Baker C, Rutledge AC, Adeli K. Fructose: a highly lipogenic nutrient implicated in insulin resistance, hepatic steatosis, and the metabolic syndrome. Am J Physiol Endocrinol Metab 2010; 299: E685-E694.

8 Ackerman Z, Oron-Herman M, Grozovski M, Rosenthal T, Pappo O, Link G et al. Fructose-induced fatty liver disease: hepatic effects of blood pressure and plasma triglyceride reduction. Hypertension 2005; 45: 1012-1018.

9 Zhang L, Perdomo G, Kim DH, Qu S, Ringquist S, Trucco M et al. Proteomic analysis of fructose-induced fatty liver in hamsters. Metabolism 2008; 57: 1115-1124.

10 Kanuri G, Spruss A, Wagnerberger S, Bischoff SC, Bergheim I. Fructose-induced steatosis in mice: role of plasminogen activator inhibitor-1, microsomal triglyceride transfer protein and NKT cells. Lab Invest 2011; 91: 885-895.

11 Spruss A, Kanuri G, Stahl C, Bischoff SC, Bergheim I. Metformin protects against the development of fructose-induced steatosis in mice: role of the intestinal barrier function. Lab Invest 2012; 92: 1020-1032.

12 Zelber-Sagi S, Nitzan-Kaluski D, Goldsmith R, Webb M, Blendis L, Halpern Z et al. Long term nutritional intake and the risk for non-alcoholic fatty liver disease (NAFLD): a population based study. J Hepatol 2007; 47: 711-717. 
13 Ouyang X, Cirillo P, Sautin Y, McCall S, Bruchette JL, Diehl AM et al. Fructose consumption as a risk factor for non-alcoholic fatty liver disease. $J$ Hepatol. 2008; 48: 993-999.

14 Abid A, Taha O, Nseir W, Farah R, Grosovski M, Assy N. Soft drink consumption is associated with fatty liver disease independent of metabolic syndrome. $J$ Hepatol 2009; 51: 918-924.

15 Higgins JPT, Green S (editors). Cochrane handbook for systematic reviews of interventions version 5.1.0 [updated march 2011]. The Cochrane Collaboration. Available from www.cochrane-handbook.org, 2011.

16 Moher D, Liberati A, Tetzlaff J, Altman DG. PRISMA Group. Preferred reporting items for systematic reviews and meta-analyses: the PRISMA statement. BMJ 2009; 339: b2535.

17 Heyland DK, Novak F, Drover JW, Jain M, Su X, Suchner U. Should immunonutrition become routine in critically ill patients? A systematic review of the evidence. JAMA 2001; 286: 944-953.

18 Elbourne DR, Altman DG, Higgins JP, Curtin F, Worthington HV, Vail A. Meta-analyses involving cross-over trials: methodological issues. Int J Epidemiol 2002; 31: 140-149.

19 Ngo Sock ET, Le KA, Ith M, Kreis R, Boesch C, Tappy L. Effects of a short-term overfeeding with fructose or glucose in healthy young males. Br J Nutr 2010; 103: 939-943.

20 Silbernagel G, Machann J, Unmuth S, Schick F, Stefan N, Häring HU et al. Effects of 4-week very-high-fructose/glucose diets on insulin sensitivity, visceral fat and intrahepatic lipids: an exploratory trial. Br J Nutr 2011; 106: 79-86.

21 Aeberli I, Gerber PA, Hochuli M, Kohler S, Haile SR, Gouni-Berthold I et al. Low to moderate sugar-sweetened beverage consumption impairs glucose and lipid metabolism and promotes inflammation in healthy young men: a randomized controlled trial. Am J Clin Nutr 2011; 94: 479-485.

22 Lê KA, Faeh D, Stettler R, Ith M, Kreis R, Vermathen P et al. A 4-wk high-fructose diet alters lipid metabolism without affecting insulin sensitivity or ectopic lipids in healthy humans. Am J Clin Nutr 2006; 84: 1374-1379.

23 Lê KA, Ith M, Kreis R, Faeh D, Bortolotti M, Tran C et al. Fructose overconsumption causes dyslipidemia and ectopic lipid deposition in healthy subjects with and without a family history of type 2 diabetes. Am J Clin Nutr 2009; 89: 1760-1765.

24 Sobrecases $\mathrm{H}$, Lê KA, Bortolotti M, Schneiter $\mathrm{P}$, Ith $\mathrm{M}$, Kreis $\mathrm{R}$ et al. Effects of short-term overfeeding with fructose, fat and fructose plus fat on plasma and hepatic lipids in healthy men. Diabetes Metab 2010; 36: 244-246.

25 Cox CL, Stanhope KL, Schwarz JM, Graham JL, Hatcher B, Griffen SC et al. Consumption of fructose- not glucose-sweetened beverages for 10 weeks increases circulating concentrations of uric acid, retinol binding protein-4, and gammaglutamyl transferase activity in overweight/obese humans. Nutr Metab 2012; 9: 68

26 Johnston RD, Stephenson MC, Grossland H, Cordon SM, Palcidi E, Cox EF et al. No difference between high-fructose and high-glucose diets on liver triacylglycerol or biochemistry in healthy overweight men. Gastroenterology 2013; 145: 1016-1025.e2.

27 Lustig RH. Fructose: metabolic, hedonic, and societal parallels with ethanol. J Am Diet Assoc. 2010; 110: 1307-1321.

28 Sievenpiper JL, de Souza RJ, Kendall CW, Jenkins DJ. Is fructose a story of mice but not men? J Am Diet Assoc 2011; 111: 219-220.

29 Sun SZ, Empie M. Fructose metabolism in humans-what isotopic tracer studies tell us. Nutr Metab (Lond) 2012; 9: 89.

30 Tappy L, Lê KA. Metabolic effects of fructose and the worldwide increase in obesity. Physiol Rev 2010; 90: 23-46.
31 Kechagias S, Ernersson A, Dahlqvist O, Lundberg P, Lindström T, Nystrom FH et al. Fast-food-based hyper-alimentation can induce rapid and profound elevation of serum alanine aminotransferase in healthy subjects. Gut 2008; 57: 649-654.

32 Musso G, Cassader M, Rosina F, Gambino R. Impact of current treatments on liver disease, glucose metabolism and cardiovascular risk in non-alcoholic fatty liver disease (NAFLD): a systematic review and meta-analysis of randomized trials. Diabetologia 2012; 55: 885-904.

33 Sievenpiper JL, de Souza RJ, Mirrahimi A, Yu ME, Carleton AJ, Beyene J et al. Effect of fructose on body weight in controlled feeding trials: a systematic review and meta-analysis. Ann Intern Med 2012; 156: 291-304.

34 Wang DD, Sievenpiper JL, de Souza RJ, Chiavaroli L, Ha V, Cozma Al et al. The effects of fructose intake on serum uric acid vary among controlled dietary trials. J Nutr 2012; 142: 916-923.

35 Livesey G, Taylor R. Fructose consumption and consequences for glycation, plasma triacylglycerol, and body weight: meta-analyses and meta-regression models of intervention studies. Am J Clin Nutr 2008; 88: 1419-1437.

36 Sievenpiper JL, Carleton AJ, Chatha S, Jiang HY, de Souza RJ, Beyene J et al. Heterogeneous effects of fructose on blood lipids in individuals with type 2 diabetes: systematic review and meta-analysis of experimental trials in humans. Diabetes Care 2009; 32: 1930-1937.

37 Ha V, Sievenpiper JL, de Souza RJ, Chiavaroli L, Wang DD, Cozma Al et al. Effect of fructose on blood pressure: a systematic review and meta-analysis of controlled feeding trials. Hypertension 2012; 59: 787-795.

38 Szczepaniak LS, Nurenberg P, Leonard D, Browning JD, Reingold JS, Grundy S et al. Magnetic resonance spectroscopy to measure hepatic triglyceride content: prevalence of hepatic steatosis in the general population. Am J Physiol Endocrinol Metab 2005; 288: E462-E468.

39 Abdelmalek MF, Suzuki A, Guy C, Unalp-Arida A, Colvin R, Johnson RJ et al. Nonalcoholic steatohepatitis clinical research network. Increased fructose consumption is associated with fibrosis severity in patients with nonalcoholic fatty liver disease. Hepatology 2010; 51: 1961-1971.

40 Marriott BP, Cole N, Lee E. National estimates of dietary fructose intake increased from 1977 to 2004 in the United States. J Nutr 2009; 139: 1228S-1235S

41 Mozaffarian D, Hao T, Rimm EB, Willett WC, Hu FB. Changes in diet and lifestyle and long-term weight gain in women and men. N Engl J Med 2011; 364: 2392-2404.

42 Ye EQ, Chacko SA, Chou EL, Kugizaki M, Liu S. Greater whole-grain intake is associated with lower risk of type 2 diabetes, cardiovascular disease, and weight gain. J Nutr 2012; 142: 1304-1313.

43 Jenkins DJ, Srichaikul K, Kendall CW, Sievenpiper JL, Abdulnour S, Mirrahimi A et al. The relation of low glycaemic index fruit consumption to glycaemic control and risk factors for coronary heart disease in type 2 diabetes. Diabetologia 2011; 54: 271-279.

44 Madero M, Arriaga JC, Jalal D, Rivard C, McFann K, Pérez-Méndez O et al. The effect of two energy-restricted diets, a low-fructose diet versus a moderate natural fructose diet, on weight loss and metabolic syndrome parameters: a randomized controlled trial. Metabolism 2011; 60: 1551-1559.

(c) (i) (2) This work is licensed under a Creative Commons AttributionNonCommercial-ShareAlike 3.0 Unported License. To view a copy of this license, visit http://creativecommons.org/licenses/by-nc-sa/3.0/

Supplementary Information accompanies this paper on European Journal of Clinical Nutrition website (http://www.nature.com/ejcn) 